\title{
Influencia de los parámetros de electrohilado en la morfología y tamaño de fibras de fibroína de seda
}

\section{Influence of electrospinning parameters on morphology and size of silk fibroin fibers}

\author{
Ana Gaviria ${ }^{1}$, Laura Lotero ${ }^{2}$, \\ Natalia Jaramillo-Quiceno ${ }^{1}$, Adriana Restrepo-Osorio ${ }^{1}$
}

\footnotetext{
${ }^{1}$ Universidad Pontificia Bolivariana, Escuela de Ingenierías, Grupo de Investigación Sobre Nuevos Materiales, Semillero de Investigación en Textiles, Medellín, Antioquia, Colombia.

${ }^{2}$ Universidad Pontificia Bolivariana, Escuela de Ingenierías, Grupo de Investigación en Sistemas Aplicados a la Industria, Medellín, Antioquia, Colombia.

e-mail: adriana.restrepo@upb.edu.co
}

\begin{abstract}
RESUMEN
El procesamiento de fibroína de seda (SF) por medio de la técnica de electrohilatura ha abierto un amplio campo de posibilidades para generar membranas fibrilares a escala micro y nanométrica, que aunados a las propiedades de biocompatibilidad y destacadas propiedades mecánicas de esta proteína, potencian su uso en diversas aplicaciones ingenieriles y biomédicas. Los procesos de electrohilatura se encuentran sujetos a una gran cantidad de variables y a las interacciones entre ellas, lo cual a su vez repercute en las propiedades morfológicas del material obtenido. En el presente trabajo se empleó SF proveniente de residuos serícolas colombianos, disuelta en ácido fórmico (AF) al $10 \% \mathrm{w} / \mathrm{w}$, se estudiaron dos condiciones de campo eléctrico $(\mathrm{R}=1$ y $2 \mathrm{kV} / \mathrm{cm})$ a tres distancias $(8,10$ y $12 \mathrm{~cm})$, y bajo dos condiciones de flujo $(0,05$ y $0.1 \mathrm{~mL} / \mathrm{h})$. Las membranas fibrosas obtenidas fueron caracterizadas por microscopía electrónica de barrido (SEM), las imágenes se analizaron con el software ImajeJ para establecer presencia de defectos tipo beads, sus diámetros y distribuciones. Se encontró que el campo eléctrico menor $\mathrm{R}=1$ disminuyó la aparición de defectos. Se realizó un análisis de varianza (ANOVA) bajo dicha condición y se hizo la comparación de medias usando la prueba Tukey $(\mathrm{P}<0.05)$ para las variables que mostraron diferencias significativas, usando el software estadístico R. Los resultados indican que los parámetros con efecto significativo son el flujo y la distancia. Por tanto, se estableció que, entre las variables estudiadas, los resultados conseguidos con $\mathrm{R}=1 \mathrm{kV} / \mathrm{cm}$, distancia de $12 \mathrm{~cm}$ y flujo de $0,1 \mathrm{~mL} / \mathrm{h}$, promovieron la obtención de membranas fibrilares con diámetros nanométricos uniformes y sin defectos tipo beads.
\end{abstract}

Palabras clave: Electrohilado, Fibroína de Seda, ANOVA, SEM.

\begin{abstract}
The processing of silk fibroin (SF) by means of the electrospinning technique has opened a wide field of possibilities to generate fibrillar membranes at micro and nanometric scale, which together with the biocompatibility properties and outstanding mechanical properties of this protein, enhance its use in various engineering and biomedical applications. The electrospinning processes are subject to a large number of variables and the interactions between them, which in turn affects the morphological properties of the material obtained. In the present work we used SF from Colombian sericulture residues, dissolved in formic acid at $10 \% \mathrm{w} / \mathrm{w}$, two electric field conditions were studied $(\mathrm{R}=1$ and $2 \mathrm{kV} / \mathrm{cm})$ at three distances $(8,10$ and $12 \mathrm{~cm})$ and under two flow conditions $(0.05$ and $0.1 \mathrm{~mL} / \mathrm{h})$. The fibrous membranes obtained were characterized by scanning electron microscopy (SEM); the images were analyzed with the ImajeJ software to establish the presence of beads defects, their diameters, and distributions. We found that the minor electric field $\mathrm{R}=1$ decreased the appearance of defects. An analysis of variance (ANOVA) was performed under this condition and the comparison of means was made using the Tukey test $(\mathrm{P}<0.05)$ for the variables that showed significant differences, using the statistical software $\mathrm{R}$. The results indicate that the parameters with significant effect were the flow and the distance. Therefore, it was established that, among the variables studied, the results obtained with $\mathrm{R}=1 \mathrm{kV} / \mathrm{cm}$, distance of $12 \mathrm{~cm}$ and flow of $0.1 \mathrm{~mL} / \mathrm{h}$, promoted the obtaining of fibrillar membranes with uniform nanometric diameters and without beads defects.
\end{abstract}


Keywords: Electrospinning, Silk Fibroin, ANOVA, SEM.

\section{INTRODUCCIÓN}

La SF, convencionalmente obtenida de la seda del capullo del gusano Bombyx mori (B. mori), es una proteína estructural con propiedades mecánicas superiores a las de otros biopolímeros naturales como el colágeno [1], es biocompatible, con permeabilidad al oxígeno y al vapor de agua, biodegradable y con una mínima reacción inflamatoria [2]. Lo anterior, sumado a la versatilidad para ser procesada para producir diferentes materiales como membranas electrohiladas, películas, geles y recubrimientos, entre otros, la han convertido en una proteína de gran interés científico y tecnológico para las industrias biomédica y farmacéutica [3].

De estos materiales, se destacan las membranas obtenidas por electrohilatura. Esta técnica permite producir fibras a escala micro y nanométrica, con propiedades geométricas y funcionales específicas, lo que ha permitido diversificar las aplicaciones de la SF en el área biomédica para la regeneración de tejidos y la liberación controlada de fármacos [4,5]. Existen alrededor de 30 parámetros diferentes que influyen en la transformación de la solución de polímero a fibras electrohiladas, entre estos: a) las propiedades de la solución, como viscosidad, conductividad y tensión superficial; b) parámetros de procesamiento como el campo eléctrico aplicado, la distancia entre la punta de la aguja y el colector además del flujo de la solución; y (c) parámetros ambientales como la temperatura y la humedad $[3,6]$.

Actualmente existen muchos tipos de equipos de electrohilado y configuraciones, y a pesar de que se han realizado diversos estudios para evaluar el efecto de las variables en la generación de fibras de SF electrohiladas uniformes y sin defectos, cuya fuente son los capullos $[2,3,6]$, las particularidades del presente estudio como el equipo de electrohilado homemade y la constitución heterogénea de los residuos fibrosos que se emplean como materia prima, hacen necesario establecer variables específicas de trabajo.

Por esta razón, en este estudio se analizó la influencia en la morfología y el tamaño de fibra de materiales electrohilados de SF, a partir de residuos fibrosos, mediante un arreglo experimental que evalúa el campo eléctrico (R), el flujo de alimentación de la solución y la distancia de la punta de la jeringa al colector fijo; con el fin de plantear unas condiciones de proceso que permitan la obtención de nanofibras de SF con propiedades morfológicas ajustadas a diferentes aplicaciones biomédicas o farmacéuticas.

\section{MATERIALES Y MÉTODOS}

\subsection{Extracción de Fibroína de Seda (SF)}

Los residuos de seda fueron desengomados empleando una solución acuosa de carbonato de sodio $\left(\mathrm{Na}_{2} \mathrm{CO}_{3}\right)$ Merck al 0,5 w/v, posteriormente fueron lavados y secados. La SF fue disuelta empleando una solución de bromuro de litio ( $\mathrm{LiBr}$ ) Sigma-Aldrich al 9,3 $\mathrm{M}$ a $60^{\circ} \mathrm{C}$, la cual se filtró y dializó en agua destilada para eliminar los remanentes de la sal empleada. La solución de SF fue centrifugada, microfiltrada y secada a $35^{\circ} \mathrm{C}$ durante $24 \mathrm{~h}$.

\subsection{Elaboración de membranas por electrohilado}

La SF se disolvió en ácido fórmico (FA), $\mathrm{CH}_{2} \mathrm{O}_{2}$, Merck del $98 \%$ de pureza, a una concentración del $10 \% \mathrm{w} / \mathrm{w}$. Posteriormente, las soluciones de fibroína fueron introducidas en jeringas de $5 \mathrm{~mL}$ de volumen, con una aguja metálica de $0,8 \mathrm{~mm}$ de diámetro (21G). Para el proceso de elaboración de las membranas por electrohilado, se empleó un colector fijo de placa plana recubierto con papel de aluminio (Figura 1). La distancia desde la punta de la aguja al colector, el campo eléctrico y el flujo de solución variaron de acuerdo con el arreglo experimental mostrado en la Tabla 1. Cabe aclarar que por limitaciones técnicas de capacidad máxima de la fuente que suministra el campo eléctrico $(20 \mathrm{kV})$, no se evaluó la condición de distancia $12 \mathrm{~cm}$ y R2. 


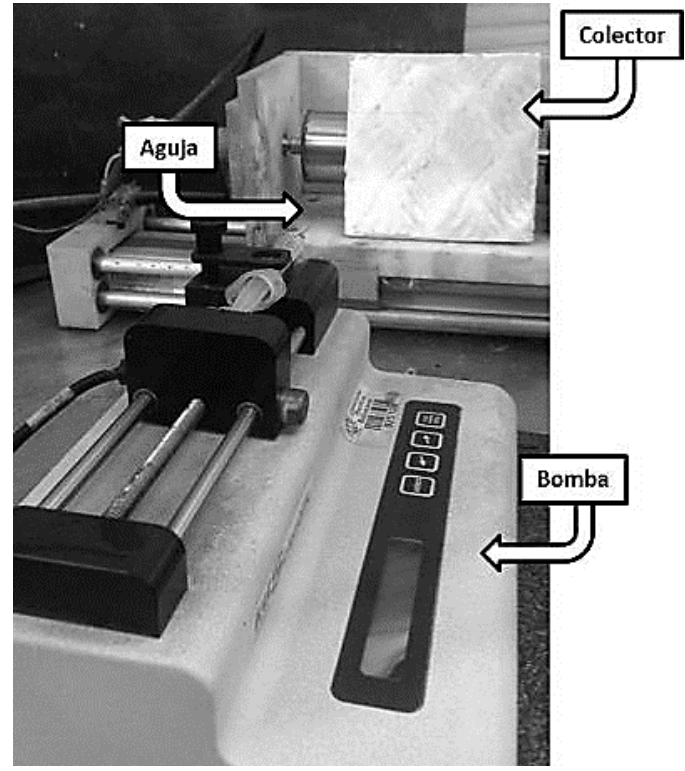

Figura 1: Equipo de electrohilado homemade

Tabla 1: Parámetros de electrohilado para las soluciones al $10 \%$ w/w SF/FA.

\begin{tabular}{|c|c|c|}
\hline DISTANCIA (cm) & CAMPO ELEECTRICO R (kV/cm) & FLUJO DE SOLUCIÓN (mL/h) \\
\hline \multirow{4}{*}{ 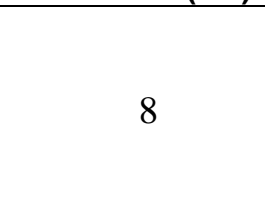 } & \multirow[t]{2}{*}{ 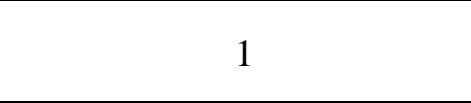 } & 0,05 \\
\hline & & 0,10 \\
\hline & \multirow{2}{*}{2} & 0,05 \\
\hline & & 0,10 \\
\hline \multirow{4}{*}{10} & \multirow{2}{*}{1} & 0,05 \\
\hline & & 0,10 \\
\hline & \multirow{2}{*}{2} & 0,05 \\
\hline & & 0,10 \\
\hline \multirow{2}{*}{12} & \multirow{2}{*}{1} & 0,05 \\
\hline & & 0,10 \\
\hline
\end{tabular}

\subsection{Caracterización morfológica}

La morfología de las membranas elaboradas por electrohilado fue analizada a partir de las imágenes obtenidas con un microscopio electrónico de barrido (SEM), JSM 6490-LV. Previo a la observación, las muestras fueron recubiertas con oro y se capturaron imágenes a 1000X y 5000X para determinar la presencia y cantidad de defectos estructurales, así como para realizar mediciones de diámetro de fibra. Para cada condición analizada se calculó el diámetro promedio a partir de 100 mediciones aleatorias utilizando el programa de análisis de imagen (ImageJ).

\subsection{Análisis estadístico}

La influencia de los parámetros de proceso individuales (campo eléctrico, flujo y distancia), así como sus interacciones, en el diámetro medio de las fibras obtenidas, fueron estudiadas por medio de un análisis de varianza (ANOVA). Para las variables que mostraron diferencias significativas se realizó comparaciones por pares de sus medias usando la prueba Tukey [7,8]. Las diferencias se consideraron estadísticamente significativas para valores $\mathrm{P}<0.05$. Para el análisis estadístico se utilizó el software $\mathrm{R}[9]$. 


\section{RESULTADOS Y DISCUSIONES}

Las imágenes SEM de las membranas electrohiladas de SF correspondientes a la relación de campo eléctrico $1 \mathrm{kV} / \mathrm{cm}(\mathrm{R} 1)$ y $2 \mathrm{kV} / \mathrm{cm}(\mathrm{R} 2)$ se presentan en la Figura 2 y Figura 3, respectivamente. Al analizar la influencia de los flujos evaluados $(0,05 \mathrm{~mL} / \mathrm{h}$ y $0,1 \mathrm{~mL} / \mathrm{h})$, se aprecia que al usar un valor menor de flujo $(0,05 \mathrm{~mL} / \mathrm{h})$ manteniendo los demás valores constantes, existe una tendencia general a la reducción de defectos tipo beads, un aumento de los diámetros de las fibras y una mayor variabilidad de estos.

Esto es comparable con lo reportado por otros autores en membranas electrohiladas de polímeros como el Nylon 6 [10] y el polivinil alcohol [11]. Por otro lado, reportes de membranas electrohiladas de SF indican que a mayores flujos se incrementan los defectos en las membranas, lo que atribuyen a dificultades en la evaporación del solvente y la caída de gotas del solvente sobre la membrana [4]. Las diferencias con los hallazgos de este trabajo pueden tener relación con que en los reportes bibliográficos se emplearon flujos mayores a los estudiados en el presente trabajo, mientras que a flujos relativamente bajos $\leq 0,1 \mathrm{~mL} / \mathrm{h}$ este comportamiento fue el opuesto. Será de interés considerar para trabajos futuros ampliar el rango de flujos evaluados, para corroborar esta hipótesis.

\begin{tabular}{|c|c|c|}
\hline $\begin{array}{l}\text { DISTAN- } \\
\text { CIA }\end{array}$ & FLUJO= 0,05 mL/h & FLUJO=0,1 mL/h \\
\hline \multicolumn{3}{|l|}{$8 \mathrm{~cm}$} \\
\hline & $\begin{array}{l}\text { A } \\
\mathrm{Dp}=184,5 \pm 11,87 \mathrm{~nm}\end{array}$ & $\begin{array}{l}\mathrm{B} \\
\mathrm{Dp}=143,8 \pm 10,2 \mathrm{~nm}\end{array}$ \\
\hline \multicolumn{3}{|l|}{$10 \mathrm{~cm}$} \\
\hline & $20 \mathrm{kV} \times 1,000 \quad 10 \mu \mathrm{m}$ & 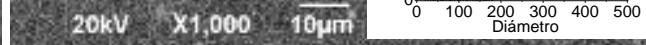 \\
\hline & $\begin{array}{l}\mathrm{C} \\
\mathrm{Dp}=114,18 \pm 6,16 \mathrm{~nm}\end{array}$ & $\begin{array}{l}\mathrm{D} \\
\mathrm{Dp}=80,99 \pm 3,18 \mathrm{~nm}\end{array}$ \\
\hline
\end{tabular}




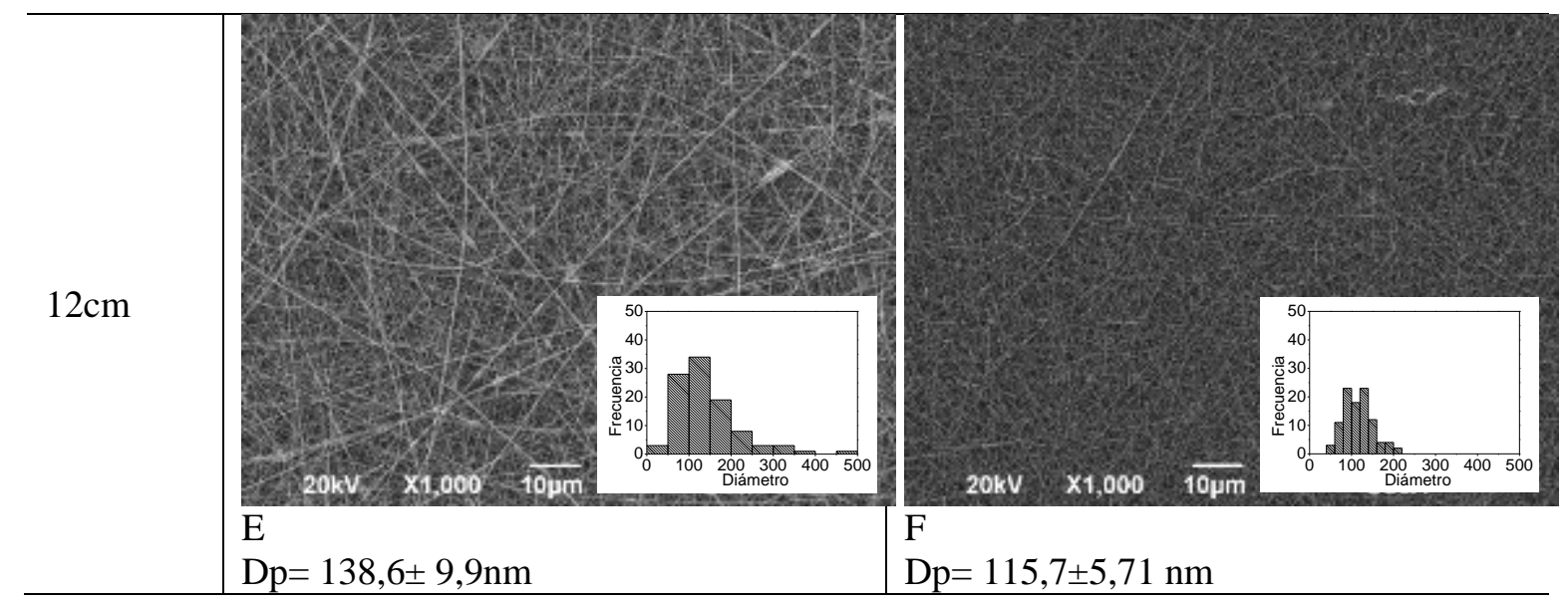

Figura 2: Imágenes SEM (1000X) para las membranas de SF obtenidas con R1

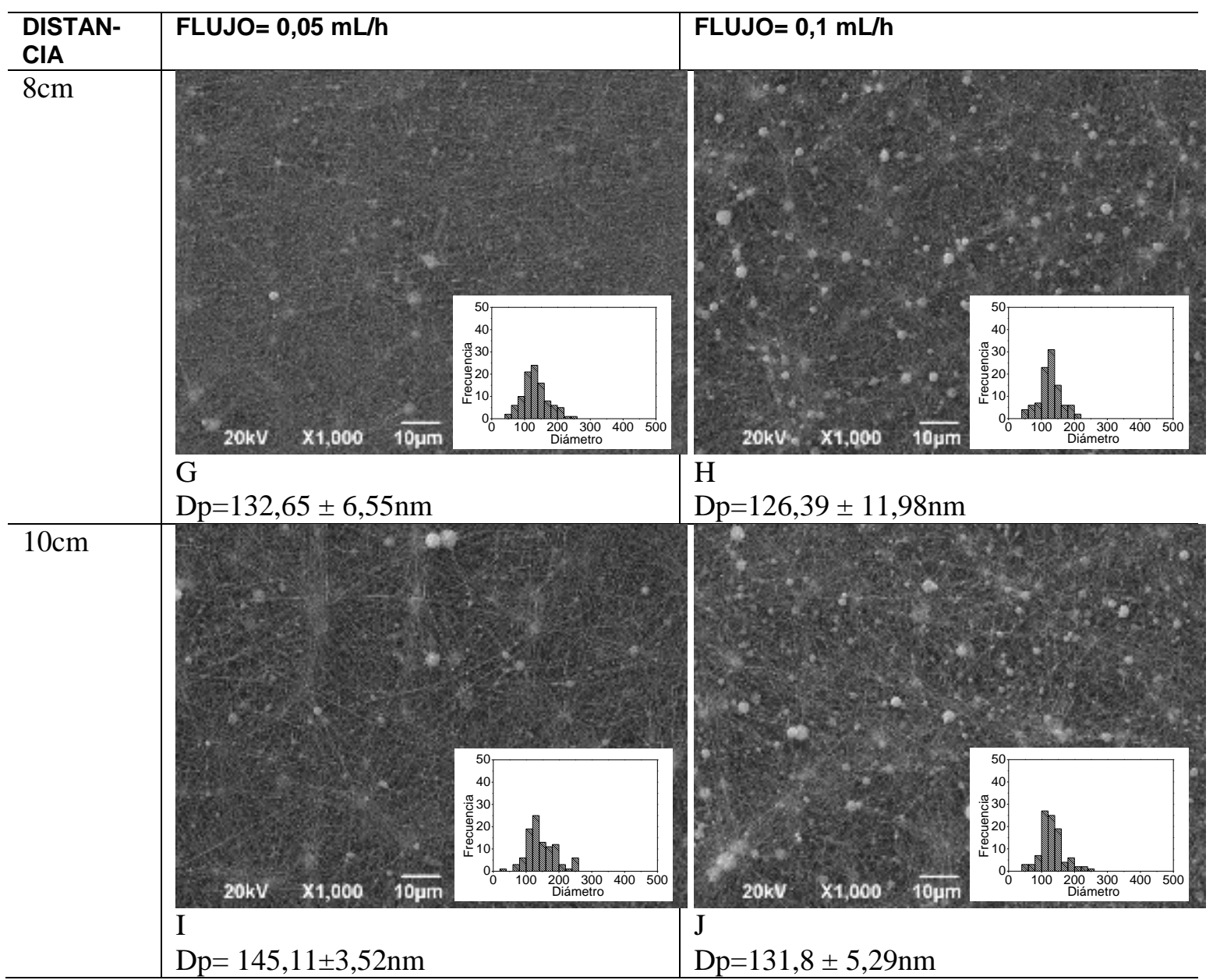

Figura 3: Imágenes SEM (1000X) para los no-tejidos obtenidos con R2.

Al analizar el efecto del campo eléctrico comparando las imágenes de la Figura 2 (R1) y la Figura 3 (R2), se observa que a mayor valor de esta variable (R2) se genera un aumento notable en la cantidad de defectos beads en comparación con las muestras obtenidas con R1. Esto se atribuye a que un aumento en el campo eléctrico interfiere con el volumen de la gota de polímero dentro del cono Taylor, reduciéndolo y provocando que la eyección de la solución polimérica sea cada vez más cercana a la punta de la aguja lo que se asocia a una mayor presencia de defectos tipo beads [12]. 
Respecto a los diámetros de las fibras obtenidas no fue posible establecer una tendencia clara atribuible al cambio en el campo eléctrico dado que una distancia de $8 \mathrm{~cm}$ los diámetros parecen tener valores menores aplicando un mayor campo eléctrico, mientras a $10 \mathrm{~cm}$ estos se incrementan. Por lo anterior, se establece que un campo eléctrico menor $(\mathrm{R}=1 \mathrm{kV} / \mathrm{cm})$, disminuye la aparición de defectos, por lo que se procede a realizar el análisis estadístico de estos datos para establecer la significancia de las variables estudiadas en función de encontrar fibras que combinen menores diámetros, mayor uniformidad y menor presencia de defectos tipo beads. Por lo tanto, se consideró pertinente reducir el ANOVA, estudiando el efecto de los parámetros distancia y flujo en la condición de campo eléctrico R1, respecto al diámetro promedio de las fibras.

En la Tabla 2 se presentan los resultados del ANOVA, a partir de los cuales se deduce que tanto el flujo como la distancia son variables que tienen un efecto significativo en el diámetro medio de las fibras obtenidas a $1 \mathrm{kV} / \mathrm{cm}$. Por su parte, la interacción entre la distancia y el flujo no resultó ser significativa.

Tabla 2: Análisis de varianza (ANOVA)

\begin{tabular}{lccccc} 
VARIABLE & GL & SSE & MSE & F & VALOR P \\
\hline Distancia & 2 & 17805 & 8903 & 125,821 & $2,63 \mathrm{e}-11$ \\
Flujo & 1 & 6255 & 6255 & 88,396 & $2,29 \mathrm{e}-8$ \\
Distancia*Flujo & 2 & 320 & 160 & 2,264 & 0,133 \\
Residuales & 18 & 1274 & 71 & & \\
\hline
\end{tabular}

En la Figura 4 se presentan los resultados de diámetro promedio obtenidos para diferentes condiciones de distancia y flujo a R1. Para ambos niveles de flujo, el aumento de la distancia produjo una disminución en el diámetro promedio, alcanzando un mínimo a los $10 \mathrm{~cm}$. Por encima de dicha distancia, los valores de diámetro promedio mostraron un leve aumento. Este comportamiento se asocia a la existencia de un punto crítico de distancia, en el que se da la formación de un jet estable y además se alcanza un tiempo de deposición suficiente para lograr la evaporación de gran parte del solvente; lo que finalmente deriva en la formación de fibras más delgadas y uniformes [3,13]. Respecto al efecto del flujo, se encontró que al aumentar esta variable se dio una reducción en los valores de diámetro promedio. De acuerdo con lo reportado por ABDELHADY et al. [14] y SCHOENMAKER et al. [15], la reducción del diámetro promedio al incrementar el flujo se atribuye a la emergencia de jets secundarios, los cuales son producto de la solidificación parcial de la solución en la punta de la jeringa, resultando en diámetros de fibra menores comparados con los del jet inicial.

De acuerdo con la Figura 4, el no-tejido obtenido a $10 \mathrm{~cm}$ y un flujo de $0,1 \mathrm{~mL} / \mathrm{h}$ permite la formación de nanofibras de menor diámetro, sin embargo según los resultados de la (D) tienen una marcada presencia de defectos tipo beads, por lo que no puede ser considerada la mejor condición evaluada. Realizando el mismo análisis, las muestras obtenidas a $10 \mathrm{~cm}-0,05 \mathrm{~mL} / \mathrm{h}$ y $12 \mathrm{~cm}-0,1 \mathrm{~mL} / \mathrm{h}$, presentan también menores diámetros (Figura 4), los resultados correspondientes a dichas muestras en la Figura 2 (C) y (F) respectivamente, indican que la muestra $10 \mathrm{~cm}-0,05 \mathrm{~mL} / \mathrm{h}$ presenta una significativa presencia de beads mientras que $12 \mathrm{~cm}$ $0,1 \mathrm{~mL} / \mathrm{h}$ no presenta este tipo de defectos, esta última muestra exhibe además una mayor uniformidad en los valores de los diámetros obtenidos, que sumado a un mayor flujo de solución favorece un proceso de producción de fibras más eficiente. 


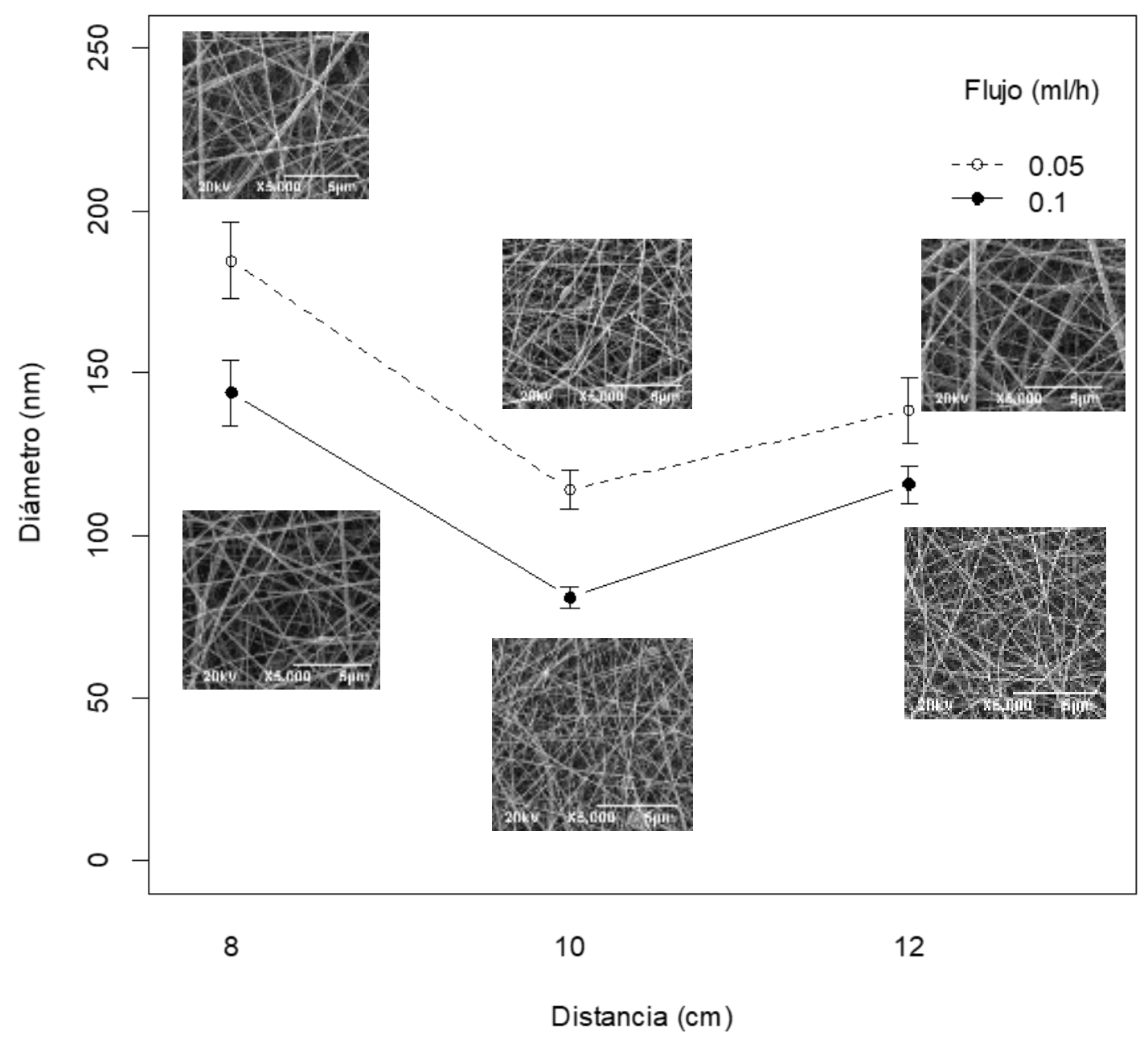

Figura 4: Diámetro promedio para diferentes condiciones de distancia y flujo.

\section{CONCLUSIONES}

En el presente trabajo se evaluaron parámetros de electrohilado de SF obtenida de residuos fibrosos disuelta en FA al $10 \%$ w/w, tales como el campo eléctrico y el flujo de la solución. Todas las condiciones evaluadas produjeron fibras a escala nanométrica con diferencias en diámetros, uniformidad y presencia de defectos tipo beads. De acuerdo con los resultados experimentales un mayor campo eléctrico y valores de menores flujo promueven la aparición de defectos tipo beads. Del análisis de estadístico de varianza se corrobora la significancia de las variables flujo y distancia, esta última en estrecha relación con el voltaje aplicado, al contenerse en el parámetro de campo eléctrico. Con lo cual, las condiciones de electrohilado, entre las estudiadas en el presente trabajo, que conduce a la obtención de los mejores resultados en cuanto a ausencia de defectos tipos beads, menores diámetros con mayor uniformidad y cantidad de fibras producidas, es la correspondiente a R1 $(1 \mathrm{kV} / \mathrm{cm})$, a una distancia de $12 \mathrm{~cm}$ y un flujo de $0,1 \mathrm{~mL} / \mathrm{h}$ partiendo de una solución de SF/FA al 10 $\% \mathrm{w} / \mathrm{w}$.

\section{AGRADECIMIENTOS}

Los autores expresan su agradecimiento a la Universidad Pontificia Bolivariana (UPB) y al CIDI por su apoyo a través del proyecto radicado 442B-08/15-18. 


\section{BIBLIOGRAFÍA}

[1] WANG D., LIU, H., FAN Y., "Silk fibroin for vascular regeneration", Microscopy Research and Technique, v. 80, n. 3, pp. 280-290, Mar. 2017.

[2] AMIRALIYAN N., NOURI, M., KISH, M.H., "Electrospinning of silk nanofibers. I. An investigation of nanofiber morphology and process optimization using response surface methodology", Fibers and Polymers, v. 10, n. 2, pp. 167-176, Apr. 2009.

[3] HAIDER, A., HAIDER, S., KANG I.K., "A comprehensive review summarizing the effect of electrospinning parameters and potential applications of nanofibers in biomedical and biotechnology", Arabian Journal of Chemistry, v. 11, n. 8, pp. 1165-1188, Dec. 2018.

[4] ALESSANDRINO, A., MARELLI, B., et al., "Electrospun Silk Fibroin Mats for Tissue Engineering", Engineering in Life Sciences, v. 8, n. 3, pp. 219-225, Jun. 2008.

[5] SUKIGARA, S., GANDHI, M., et al., "Regeneration of Bombyx mori silk by electrospinning. Part 2. Process optimization and empirical modeling using response surface methodology", Polymer., v. 45, n. 11, pp. 3701-3708, May 2004.

[6] SUKIGARA, S., GANDHI M., et al., "Regeneration of Bombyx mori silk by electrospinning - part 1: processing parameters and geometric properties", Polymer, v. 44, n. 19, pp. 5721-5727, Sep. 2003.

[7] BOLAND, E.D., COLEMAN, B.D., et al., "Electrospinning polydioxanone for biomedical applications", Acta Biomaterialia., v. 1, n. 1, pp. 115-123, Jan. 2005.

[8] VAZ, B.S, COSTA, J.A.V., et al., "Production of polymeric nanofibers with different conditions of the electrospinning process", Matéria, v. 22, n. 2, Jul. 2017.

[9] R CORE TEAM., "R: A Language and Environment for Statistical Computing." Vienna, Austria: R Foundation for Statistical Computing., 2017.

[10] ZARGHAM, S., BAZGIR, S., et al., "The Effect of Flow Rate on Morphology and Deposition Area of Electrospun Nylon 6 Nanofiber", Journal of Engineered Fibers and Fabrics, v. 7, n. 4, pp. 43-49, Dec. 2012.

[11] RODOPLU D., MUTLU, M., "Effects of electrospinning setup and process parameters on nanofiber morphology intended for the modification of quartz crystal microbalance surfaces", Journal of Engineered Fibers and Fabrics, v. 7, n. 2, pp. 118-123, 2012.

[12] SILL, T.J., VON RECUM H.A., "Electrospinning: Applications in drug delivery and tissue engineering", Biomaterials, v. 29, n. 13, pp. 1989-2006, May 2008.

[13] BHARDWAJ N., KUNDU, S.C., "Electrospinning: A fascinating fiber fabrication technique", Biotechnology Advances, v. 28, n. 3, pp. 325-347, May 2010.

[14] ABDEL-HADY F., ALZAHRANY, A. et al., "Experimental Validation of Upward Electrospinning Process", ISRN Nanotechnology., v. 2011, pp. 1-14, 2011.

[15] DE SCHOENMAKER, B., VAN DER SCHUEREN, L., CEYLAN, Ö., CLERCK, K., "Electrospun polyamide 4.6 nanofibrous nonwovens: Parameter study and characterization", Journal of Nanomaterials, v. 2012, pp. 1-9, May 2012.

\section{ORCID}

Ana Gaviria

Laura Lotero

Natalia Jaramillo-Quiceno

Adriana Restrepo-Osorio
https://orcid.org/0000-0002-9369-451X

https://orcid.org/0000-0002-6537-3276

https://orcid.org/0000-0002-8151-5465

https://orcid.org/0000-0003-3226-357X 